\title{
A Novel Approach for Discovering Retrotransposons: Characterization of a Long Terminal Repeat Element in the Spoilage Yeast Pichia membranaefaciens and Its Use in Strain Identification
}

\author{
BRUCE M. PEARSON, ANDREW T. CARTER, JUDITH M. FURZE, AND IAN N. ROBERTS* \\ National Collection of Yeast Cultures, Institute of Food Research, Norwich Research Park, \\ Colney, Norwich NR4 7UA, United Kingdom
}

\begin{abstract}
A novel PCR-based approach designed to detect retrotransposon long terminal repeat (LTR) elements via their association with tRNA genes was applied to Pichia membranaefaciens, an industrially important food spoilage yeast. A single primer based on tRNA gene sequences was used to amplify DNA fragments from different strains, and an observed fragment size difference among strains was found to correspond to the expected size of an integrated LTR. A 289-bp element was cloned as part of the larger fragment and shown to be present in a high copy number and variable genomic location in all strains examined. Sequence analysis revealed the element to be bounded by nucleotides TG at the $5^{\prime}$ end and CA at the $3^{\prime}$ end and to exhibit target site duplication and other sequence motifs diagnostic of retrotransposon LTRs. LTR sequence data enabled the development of a rapid identification method which distinguished among different strains. The novel method for LTR isolation and the strain identification system are both likely to prove generally applicable for a wide range of other organisms.
\end{abstract}

Retrotransposons, mobile genetic elements sharing some of the characteristics of retroviruses, have played an important role in genome evolution in all eukaryotes and typically constitute some 5 to $10 \%$ of the genome (5). They have been studied extensively in a number of different yeast species and have also been used in the construction of vectors for genetic transformation. More recently, they have proved valuable in the development of rapid methods to discriminate among different strains of the brewing and wine yeast Saccharomyces cerevisiae $(16,19)$. These methods depend upon polymorphisms in the genomic distribution and orientation of the long terminal repeat (LTR) element of the extensively described Ty (transposon yeast) retrotransposons (for a review, see reference 6). As well as being part of intact retrotransposons, LTRs are found in variable genomic locations as solo elements thought to arise through homologous recombination between the two LTRs of a single element and subsequent deletion of internal regions. Amplification of sequences intervening between neighboring LTRs produces strain fingerprints consisting of DNA fragments varying in size among strains.

Although many retrotransposons have been described (24), confirmation of their presence in fungal species other than $S$. cerevisiae, e.g., Cladosporium fulvum (18), Candida albicans (9), Schizosaccharomyces pombe (17), and Yarrowia lipolytica (21), has been relatively recent. Such elements have generally been discovered either serendipitously or by sequencing of medium-copy-number repetitive DNA. Methods based on degenerate PCR primers designed to amplify conserved regions of the reverse transcriptase gene have also been used to identify retrotransposons in some plant and animal species $(10,22)$, and we have recently extended this approach to yeasts (20a). However, for strain identification purposes it is preferable to obtain LTR sequences directly rather than by association with

* Corresponding author. Mailing address: NCYC, Institute of Food Research, Norwich Research Park, Colney, Norwich NR4 7UA United Kingdom. Phone: 441603 255000. Fax: 441603458414. Electronic mail address: ian.roberts@bbsrc.ac.uk. a reverse transcriptase gene, since the latter may be absent from truncated elements and even when present may be located up to $4 \mathrm{~kb}$ from the LTR.

Our approach to find such an element in Pichia membranaefaciens was therefore designed to detect LTRs present in close association with tRNA genes, known hot spots for integration of this class of transposon (7). We report here the use of a PCR primer to amplify regions corresponding to tRNA genes in a range of $P$. membranaefaciens strains, the identification of a molecular weight shift in one strain corresponding to the expected size of an integrated LTR, and the subsequent isolation of an intact LTR element present in multiple copies in the genome. We also report the sequence and structural characteristics of the element and its use in confirming the general utility of the strain identification method.

\section{MATERIALS AND METHODS}

Strains, media, and cultivation conditions. A variety of strains of $P$. membranaefaciens and related yeasts, originally isolated from a wide range of environments, were obtained from the United Kingdom National Collection of Yeast Cultures (NCYC), Norwich, and the Centraalbureau voor Schimmelcultures (CBS), Delft, The Netherlands. Details of strains and sources are given in Table 1. All strains were streaked on YM plates $(0.3 \%$ yeast extract, $0.3 \%$ malt extract, $0.5 \%$ peptone, $1 \%$ glucose $[\mathrm{pH} 5.5]$ ) and grown at $24^{\circ} \mathrm{C}$ to produce single colonies.

tRNA primer and PCR conditions. The primer 5'-TCCAGCCGGGAATC GAAC-3', designed from a conserved tRNA gene sequence from $S$. cerevisiae, was synthesized by using an Applied Biosystems model 394 DNA Synthesizer and used to amplify DNA fragments from single colonies of $P$. membranaefaciens strains.

Yeast cells taken from a single colony were suspended in $10 \mu \mathrm{l}$ of water and held on ice for $10 \mathrm{~min}$ prior to addition of $90 \mu \mathrm{l}$ of PCR buffer containing $0.2 \mathrm{mM}$ deoxynucleoside triphosphates and $1 \mu \mathrm{g}$ of primer. PCR amplification was performed by using an Omnigene thermal cycler (Hybaid, Teddington, United Kingdom). Cycling conditions were as follows: $92^{\circ} \mathrm{C}$ for 2 min with subsequent addition of $1 \mu \mathrm{l}$ of Taq polymerase (AmpliTaq; Perkin-Elmer Ltd., Beaconsfield, United Kingdom), followed immediately by amplification at $92^{\circ} \mathrm{C}$ for $2 \mathrm{~min}, 52^{\circ} \mathrm{C}$ for $3 \mathrm{~min}$, and $72^{\circ} \mathrm{C}$ for $2 \mathrm{~min}(2 \mathrm{cycles})$ and then at $90^{\circ} \mathrm{C}$ for $2 \mathrm{~min}, 52^{\circ} \mathrm{C}$ for 3 $\min$, and $72^{\circ} \mathrm{C}$ for $2 \mathrm{~min}(33 \mathrm{cycles}$ ). Aliquots $(20 \mu \mathrm{l})$ of reaction products were run on $1.5 \%$ agarose gels.

DNA manipulations. PCR-amplified DNA fragments were purified from lowmelting-point agarose gels by using PCR Wizard (Promega, Southampton, 
TABLE 1. Yeast strains used in this study

\begin{tabular}{|c|c|c|}
\hline Strain & Species & Source \\
\hline $\operatorname{CBS} 107^{\mathrm{T}}$ & Pichia membranaefaciens & Type strain \\
\hline NCYC21 & Pichia membranaefaciens & Brewery \\
\hline NCYC51 & Pichia membranaefaciens & Soil \\
\hline NCYC52 & Pichia membranaefaciens & Grape must \\
\hline NCYC55 & Pichia membranaefaciens & Calliphora $\mathrm{sp}$. \\
\hline NCYC169 & Pichia membranaefaciens & Grape must \\
\hline NCYC330 & Pichia membranaefaciens & Draught beer \\
\hline NCYC333 & Pichia membranaefaciens & Draught beer \\
\hline NCYC542 & Pichia membranaefaciens & Slime flux \\
\hline NCYC714 & Pichia membranaefaciens & Lemonade \\
\hline NCYC715 & Pichia membranaefaciens & Lemonade \\
\hline NCYC795 & Pichia membranaefaciens & Seawater \\
\hline CBS598 & Pichia membranaefaciens & Lambic beer \\
\hline CBS638 & Pichia membranaefaciens & Wine \\
\hline CBS5516 & Pichia membranaefaciens & Soil \\
\hline NCYC116 & Candida valida & Bees wine \\
\hline NCYC327 & Candida valida & Draught beer \\
\hline NCYC339 & Candida valida & Draught beer \\
\hline NCYC1575 & Candida valida & Fodder yeast \\
\hline NCYC432 & Pichia anomala & Type strain \\
\hline $\mathrm{CBS} 926^{\mathrm{T}}$ & Pichia cactophila & Type strain \\
\hline $\mathrm{NCYC175^{ \textrm {T } }}$ & Pichia pastoris & Type strain \\
\hline $\mathrm{NCYC6}^{\mathrm{T}}$ & Candida pseudotropicalis & Type strain \\
\hline NCYC473 & Geotrichum capitatum & Cattle \\
\hline NCYC412 & Kluyveromyces thermotolerans & Brewery \\
\hline NCYC956 & Saccharomyces cerevisiae & Wild yeast \\
\hline NCYC691 ${ }^{\mathrm{T}}$ & Saccharomycopsis fibuligera & Type strain \\
\hline NCYC1427 & Zygosaccharomyces bailii & $\begin{array}{l}\text { Food } \\
\text { spoilage }\end{array}$ \\
\hline NCYC1495 & Zygosaccharomyces bisporus & Type strain \\
\hline NCYC568 & Zygosaccharomyces rouxii & Type strain \\
\hline
\end{tabular}

United Kingdom) according to the manufacturer's instructions. Isolated fragments were cloned by ligation into vector $\mathrm{pCR}^{\mathrm{TM}} \mathrm{II}$ (TA Cloning Kit; Invitrogen Corporation, San Diego, Calif.) and sequenced with Sequenase (U.S. Biochemicals, Ltd., Cleveland, Ohio) according to the manufacturer's instructions.

Genomic DNA was prepared from yeast cells grown in YM broth cultures at $24^{\circ} \mathrm{C}$. DNA was digested with $S s p \mathrm{I}$, and genomic blots were probed with the LTR by using the ECL Direct Labelling Kit (Amersham, Little Chalfont, United Kingdom) as described previously (8).

LTR primers for rapid strain identification. The following primers were designed by using LTR sequence data: FP1, 5'-CTAATATTCTCAAACATAA ATACAA-3'; FP2, 5'-CAATGGATGTTCATAATAGAA-3'; JF1, 5'-TCATGA TTGGCTTTGTCATC-3'; and JF2, 5'-CGAGGGATAAGAGATAAGAC-3'. These primers were used in various combinations in multiplex PCR assays with an Omnigene thermal cycler (Hybaid). Oligonucleotide synthesis, the PCR mixture, and cycling conditions were identical to those used for the tRNA primer (see above).

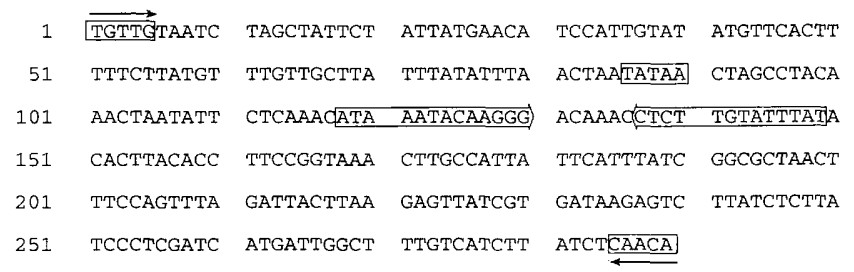

FIG. 2. Sequence and structural characteristics of the LTR. Flanking inverted repeats are shown boxed and arrowed. A putative TATA box and internal inverted repeats (arrowed boxes) are also indicated.

Nucleotide sequence analysis and accession number. DNA sequences were analyzed by using the Genetics Computer Group (University of Wisconsin) suite of programs. The $P$. membranaefaciens NCYC 21 LTR sequence plus flanking sequences have been submitted to the EMBL database in Heidelberg, Germany (accession number X82031). The sequence of the unoccupied target region from $P$. membranaefaciens CBS $107^{\mathrm{T}}$ has also been submitted (accession number $\mathrm{X} 82026)$.

\section{RESULTS}

Detection, cloning, and sequence analysis. Amplification products from different $P$. membranaefaciens strains following PCR with the tRNA primer included a fragment of approximately $500 \mathrm{bp}$ in all strains except NCYC 21 and NCYC 51. In NCYC 51 two additional fragments were detected, but in NCYC 21 the 500-bp fragment was replaced by one other fragment of approximately $800 \mathrm{bp}$, indicating an increase in size corresponding to the expected size of an LTR element. NCYC 21 was therefore selected for further study. Both 500and 800-bp fragments were cloned and sequenced. Sequence analysis revealed an integrated element of 289 bp (Fig. 1) with structural characteristics and sequence motifs characteristic of retrotransposon LTRs (Fig. 2). We have designated this element Pm-T1.

Hybridization analysis. The Pm-T1 LTR element was found by hybridization analysis to be present in multiple copies, with the copy number and genomic location varying among different strains of $P$. membranaefaciens (Fig. 3). Hybridization analysis also indicated the element to be present in strains of Candida valida, the anamorph (asexual form) of $P$. membranaefaciens, but absent from all other yeast species examined (Table 1).

Rapid strain identification. Four primers (see Materials and Methods) designed by using the LTR sequence were found to amplify fragments of the genome with sizes varying depending

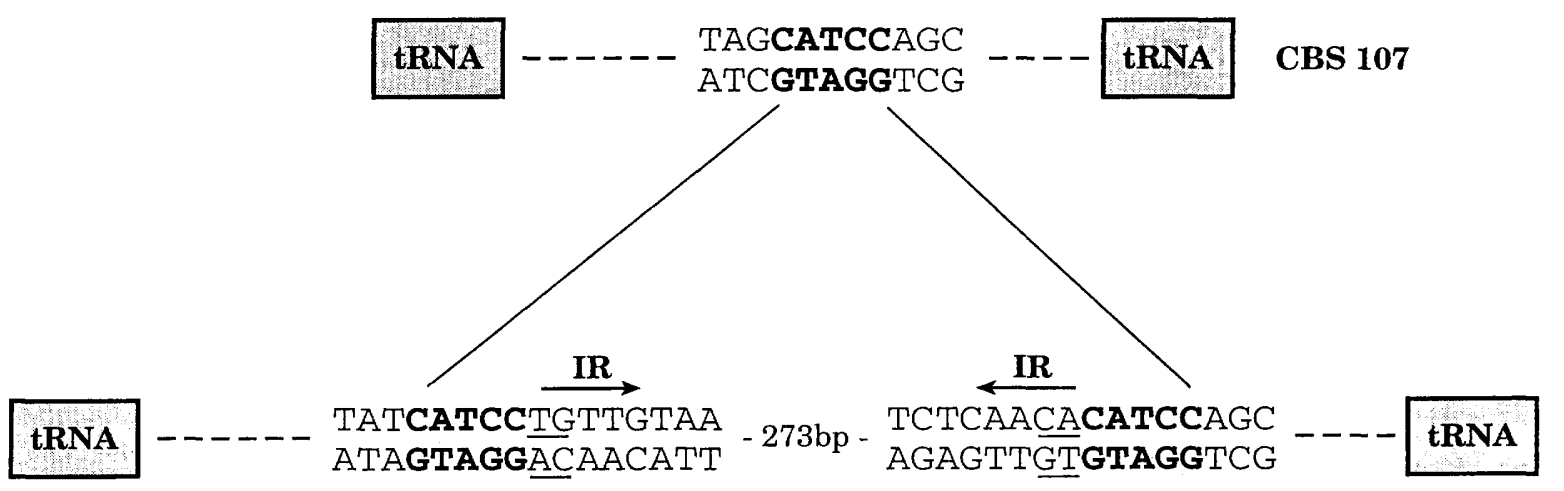

NCYC 21

FIG. 1. Sequence comparison of PCR-amplified tRNA gene regions in two strains of $P$. membranaefaciens. NCYC 21 shows a 289 -bp insert and a target site duplication (boldface type) relative to the type strain, CBS 107. The insert is bounded by TG at the $5^{\prime}$ end and CA at the $3^{\prime}$ end (underlined) and a 5-bp inverted repeat (IR) (arrows). 


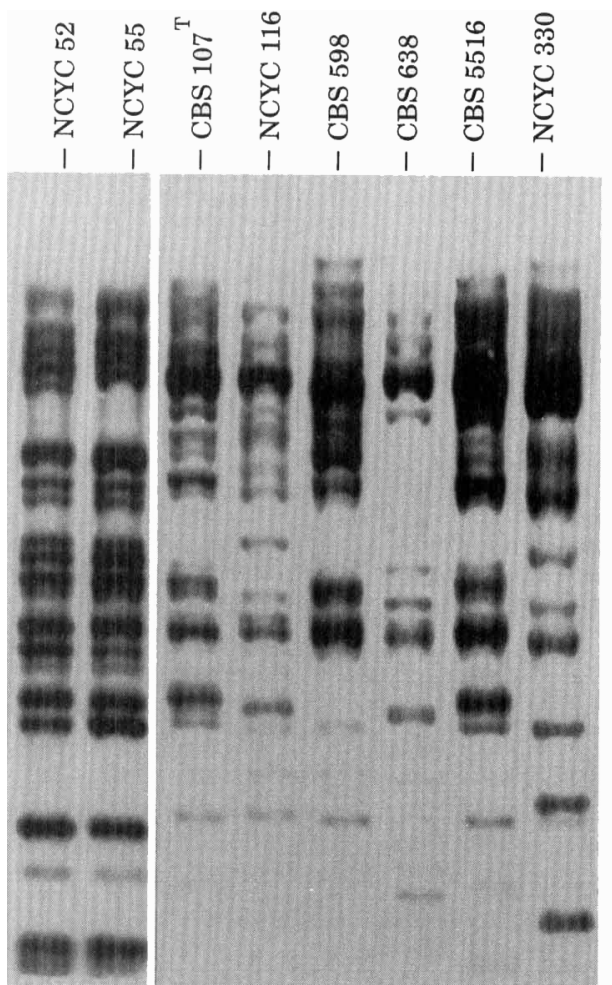

FIG. 3. Genomic DNA of $P$. membranaefaciens strains digested with $S s p \mathrm{I}$ and probed with the LTR.

on the combination of primers used. The most discriminatory fingerprint was obtained by combining the three primers FP1, FP2, and JF1 (Fig. 4).

\section{DISCUSSION}

Rapid methods for identification of strains of food spoilage yeasts are becoming increasingly important in a range of industrial contexts. We therefore sought to extend a previously described method for strain identification of the brewing yeast $S$. cerevisiae (19) in order to examine its usefulness in discriminating among strains of food spoilage yeasts. To achieve this, a novel approach to identify retrotransposons corresponding to the Ty element in species of yeasts other than $S$. cerevisiae was required.

A method which avoided the need for the large-scale screening and sequencing implicit in other approaches was designed. Direct amplification of LTRs associated with tRNA genes was found to be possible, as indicated by fragment size differences among strains of $P$. membranaefaciens. Since LTR retrotransposons are a ubiquitous class of elements which integrate preferentially near tRNA genes in all eukaryotes, the approach should be applicable to many organisms, including other yeasts. Although amplification of entire inserted retrotransposons is not possible by conventional PCR because of the large size of the resulting fragment, recent refinements (2) can achieve amplification of fragments of the required size. Therefore, an extra dimension to retrotransposon discovery by this approach may be added in the near future.

By the method described above, an LTR associated with two tRNA genes in $P$. membranaefaciens ( $P m$-T1) was successfully cloned. In common with all known LTRs, the $P$. membranaefaciens element is bounded by TG at the $5^{\prime}$ end and CA at the

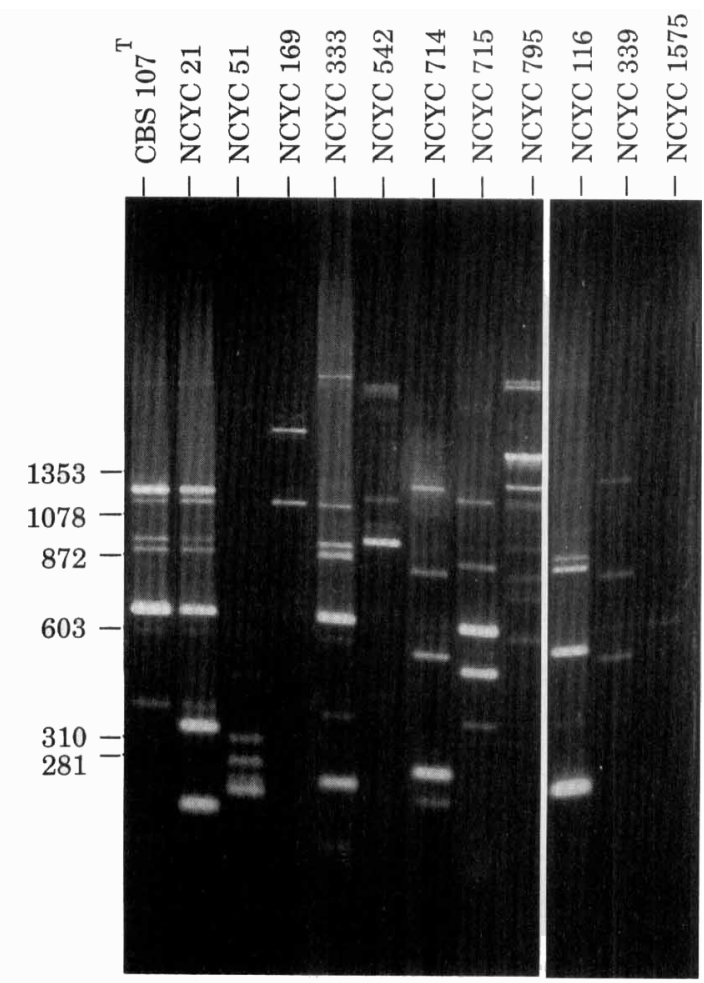

FIG. 4. PCR fingerprints of $P$. membranaefaciens and $C$. valida strains obtained by using LTR sequence primers FP1, FP2, and JF1. Numbers on the left indicate base pairs.

$3^{\prime}$ end and exhibits a 5-bp target site duplication. In addition, putative TATA box and termination signals are present in positions predicted by reference to other LTRs. The element also shows internal inverted repeats. As expected, the LTR was found to be present in a high copy number, with both copy number and genomic location varying among strains. The last two properties enabled the development of a rapid, PCRbased strain identification method for $P$. membranaefaciens.

Strain identification systems based on PCR offer many advantages in terms of speed and simplicity, with considerable savings in labor over conventional tests. The method we have described is likely to represent a reliable test and will also find application in determining sources of spoilage, e.g., when two or more strains of different origin are suspected. Strain identification systems based on transposable element profiles have also been proved reliable for prokaryotes (1).

The ability to rapidly characterize yeasts on the basis of endogenous retrotransposons may also contribute to the identification of these organisms. Yeast taxonomy has for many years been the subject of some controversy among specialists (3). Conventional methods of yeast taxonomy are based on a range of morphological and biochemical characteristics (14). However, since many such phenotypic characters are absent or inherently variable in yeasts, a large number of time-consuming and labor-intensive tests may be required for accurate identification of some strains (4). Recently, analysis of smallsubunit rRNA gene sequences has revolutionized the classifcation and identification of bacteria (23) and proved valuable for phylogenetic studies of yeasts (15). The establishment of genetic interrelationships among different species of yeasts by this method is now routine (11-13). The rRNA molecule has a mosaic structure with slowly evolving, highly conserved regions interspersed with more rapidly evolving and variable regions, 
e.g., the internal transcribed spacer (ITS) regions (20), which have provided valuable signature sequences for species and strain delimitation. However, for very closely related strains other methods of identification, e.g., electrophoretic karyotyping, are required. The method described above offers a rapid additional, complementary approach to meeting this requirement and may also provide information useful in studies of yeast evolution, which, in common with that of other eukaryotes, will have been markedly influenced by retrotransposition.

\section{ACKNOWLEDGMENTS}

We are grateful to the EC Agricultural Industrial Research Program (contract CT93-0830) for support.

We thank M. D. Collins for critically reading the manuscript.

\section{REFERENCES}

1. Baquar, N., E. J. Threlfall, B. Rowe, and J. Stanley. 1993. Molecular subtyping with a single Salmonella-typhimurium phage type, DT204C, with a PCR-generated probe for IS200. FEMS Lett. 112:217-222.

2. Barnes, W. M. 1994. PCR amplification of up to 35-kb DNA with high fidelity and high yield from lambda bacteriophage templates. Proc. Natl. Acad. Sci. USA 91:2216-2220.

3. Barnett, J. A. 1992. The taxonomy of the genus Saccharomyces Meyen ex Reess-a short review for non-taxonomists. Yeast 8:1-23.

4. Barnett, J. A., R. W. Payne, and D. Yarrow. 1990. Yeasts: characteristics and identification. Cambridge University Press, Cambridge.

5. Berg, D. E., and M. M. Howe (ed.). 1989. Mobile DNA. American Society for Microbiology, Washington, D.C.

6. Boeke, J. D., and S. B. Sandmeyer. 1991. Yeast transposable elements, p. 193-261. In J. R. Broach, J. R. Pringle, and E. W. Jones (ed.), The molecular biology of the yeast Saccharomyces, vol. 1. Genome dynamics, protein synthesis, and energetics. Cold Spring Harbor Laboratory Press, Cold Spring Harbor, N.Y.

7. Brodeur, G. M., S. B. Sandmeyer, and M. V. Olsen. 1983. Consistent association between sigma elements and tRNA genes in yeast. Proc. Natl. Acad. Sci. USA 80:3292-3296.

8. Carter, A. T., A. Narbad, B. M. Pearson, K.-F. Beck, B. Baum, M. Logghe, R. Contreras, and M. Schweizer. 1994. Phosphoribosylpyrophosphate synthetase (PRS): a new gene family in Saccharomyces cerevisiae. Yeast 10: 1031-1044.
9. Chen, J.-Y., and W. A. Fonzi. 1992. A temperature regulated, retrotransposon-like element from Candida albicans. J. Bacteriol. 174:5624-5632.

10. Flavell, A. J., E. Dunbar, R. Anderson, S. R. Pearce, R. Hartley, and A Kumar. 1992. Ty1-copia retrotransposons are ubiquitous and heterogenous in higher plants. Nucleic Acids Res. 20:3639-3644.

11. Hendriks, L., A. Goris, Y. Vanderpeer, J. M. Neefs, M. Vancanneyt, K. Kersters, J. F. Berny, G. L. Hennebert, and R. Dewachter. 1992. Phylogenetic-relationships among ascomycetes and ascomycete-like yeasts as deduced from small ribosomal-subunit RNA sequences. Syst. Appl. Microbiol. 15:98-104.

12. James, S. A., M. D. Collins, and I. N. Roberts. 1994. Genetic interrelationships among species of the genus Zygosaccharomyces as revealed by small sub-unit rRNA gene sequences. Yeast 10:871-881.

13. James, S. A., M. D. Collins, and I. N. Roberts. 1994. The genetic relationship of Lodderomyces elongisporus to other ascomycete yeast species as revealed by small sub-unit rRNA gene sequences. Lett. Appl. Microbiol. 19:308-311.

14. Kreger-van Rij, N. J. W. (ed.). 1984. The yeasts: a taxonomic study, 3rd ed. Elsevier Science Publishers B. V., Amsterdam.

15. Kurtzman, C. 1992. rRNA sequence comparison for assessing phylogenetic relationships among yeasts. Int. J. Syst. Bacteriol. 42:1-6.

16. Lavallee, F., Y. Salvas, S. Lamy, D. Y. Thomas, R. Degre, and L. Dulau. 1994. PCR and DNA fingerprinting used as quality control in the production of wine yeast strains. Am. J. Enol. Vitic. 45:86-91.

17. Levin, H. L., D. C. Weaver, and J. D. Boeke. 1990. Two related families of retrotransposons from Schizosaccharomyces pombe. Mol. Cell. Biol. 10:67916798.

18. McHale, M. T., I. N. Roberts, S. M. Noble, C. Beaumont, M. P. Whitehead, D. Seth, and R. P. Oliver. 1992. Cf-T1, an LTR retrotransposon in Cladosporium fulvum, a fungal pathogen of tomato. Mol. Gen. Genet. 233:337-347.

19. Ness, F, F. Lavallee, D. Dubourdieu, M. Aigle, and L. Dulau. 1993. Identification of yeast strains using the polymerase chain reaction. J. Sci. Food Agric. 62:89-94.

20. Olsen, G. J., C. R. Woese, and R. Overbeek. 1994. The winds of (evolutionary) change: breathing new life into microbiology. J. Bacteriol. 176:1-6.

20a.Roberts, I. N., and J. M. Furze. Unpublished data.

21. Schmid-Berger, N., B. Schmid, and G. Barth. 1994. Ylt1, a highly repetitive retrotransposon in the genome of the dimorphic fungus Yarrowia lipolytica. J. Bacteriol. 176:2477-2482.

22. Wichman, H. A., and R. A. Van Den Bussche. 1992. In search of retrotransposons: exploring the potential of the PCR. BioTechniques 13:258-263.

23. Woese, C. R. 1987. Bacterial evolution. Microbiol. Rev. 51:221-271.

24. Xiong, Y., and T. H. Eickbush. 1990 . Origin and evolution of retroelements based upon their reverse transcriptase sequences. EMBO J. 9:3353-3362. 